\title{
Micro(glial)-managing executive function: white matter inflammation drives catatonia
}

\author{
Sarah E. Pease-Raissi and Jonah R. Chan \\ Department of Neurology and UCSF Weill Institute for Neuroscience, UCSF, San Francisco, California, USA.
}

\begin{abstract}
White matter abnormalities are prevalent in neuropsychiatric disorders such as schizophrenia, but it is unclear whether these abnormalities represent a cause or consequence of these disorders. Reduced levels of the myelin protein 2'-3'-cyclic nucleotide $3^{\prime}$-phosphodiesterase (CNP) are associated with the schizophrenic symptom catatonia in both humans and mouse models. In this issue of the $J C l$, Janova et al. show that reduced CNP levels correlate with catatonia and white matter inflammation in human subjects. Furthermore, they demonstrate that microglial ablation prevents and alleviates catatonic signs in $\mathrm{Cnp}^{-/-}$mice, indicating that microglialmediated inflammation causes catatonia. Together, this study identifies a cellular mechanism by which subtle myelin abnormalities cause low-grade neuroinflammation and catatonic behavior.
\end{abstract}

\section{Introduction}

Myelin sheaths are lipid-rich membrane extensions generated by oligodendrocytes that wrap around axons. These extensions enable rapid action potential conduction and provide metabolic support to axons (1). Myelinated axons bundled into white matter tracts provide intracortical and cortico-subcortical connectivity in the CNS. White matter abnormalities are associated with neuropsychiatric disorders, such as schizophrenia, depression, and autism (24). However, it is unclear whether and how such abnormalities directly contribute to disease-related phenotypes.

Deficits in the oligodendrocytespecific myelin protein 2'-3'-cyclic nucleotide 3 '-phosphodiesterase (CNP) in mice and humans provide insight into the contribution of myelin abnormalities to psychiatric disease. CNP is reduced in postmortem brains of schizophrenic subjects (5-7). The CNP SNP rs2070106-AA, which results in lower CNP expression (7-9), correlates with a catatonia-depres- sion syndrome in human schizophrenic subjects (10). Catatonia, a neuropsychiatric symptom often associated with schizophrenia, reflects a temporary impairment of executive function and manifests as episodes of immobility, stupor, or purposeless overactivity. Schizophrenic subjects with the rs2070106-AA genotype also show diffusion tensor imaging indicators of axonal loss/degeneration in the major interhemispheric white matter tract, the corpus callosum (10).

In mice lacking $\mathrm{CNP}\left(\mathrm{Cnp}^{-/-}\right)$, oligodendrocytes form structurally normal myelin, but have a subtle myelin abnormality that results in axon degeneration and neuroinflammation (11). Aging $\mathrm{Cnp}^{-/-}$mice eventually develop gross motor deficits due to axon loss and exhibit early mortality (11). Mice heterozygous for CNP $\left(\mathrm{Cnp}^{+/}\right)$have about a $50 \%$ reduction of $\mathrm{CNP}$ and exhibit very late-onset neuroinflammation and axon degeneration, but lack gross motor deficits (10). The low-grade neuroinflammation seen in $\mathrm{Cnp}^{-/-}$and aged $\mathrm{Cnp}^{+/-}$mice

Related Article: p. 734

Conflict of interest: J.R. Chan is cofounder of Inception 5 and a consultant for Inception Sciences. S.E. Pease-Raissi and J.R. Chan declare research support from Mead Johnson Nutrition.

Reference information: / Clin Invest. 2018;128(2):564-566. https://doi.org/10.1172/JCI98761.

is characterized by recruitment of both the innate immune system, involving microglial activation and astrocytosis, and the adaptive immune system, involving $\mathrm{T}$ cell infiltration (10-12). Aged $\mathrm{Cnp}^{+-}$mice exhibit several behavioral phenotypes, notably increased depressive and catatonic behavior that is analogous to the catatonia-depression syndrome in schizophrenic patients carrying rs2070106-AA (10). Interestingly, mice heterozygous for another myelin protein, myelin basic protein (MBP), also exhibit neuroinflammation and late-onset catatonia, although neurodegeneration is not observed in these animals (13). In this issue, Janova and colleagues (14) hypothesized that minor myelin abnormalities cause white matter inflammation and thereby drive catatonic behavior.

\section{Microglia ablation treats} myelin-associated catatonia

As reduced levels of CNP are associated with catatonic behavior and neuroinflammation in both mice and humans, Janova et al. asked whether microglial-mediated white matter inflammation underlies catatonia in human subjects and mouse models (14). Using two independent data sets of schizophrenic human subjects, Janova et al. found that schizophrenic subjects have a high prevalence of catatonic signs and show increased symptom severity with age. Presence of the CNP loss-of-function SNP rs2070106-AA correlated with increased severity of catatonic signs in schizophrenic patients. Furthermore, MRI scans of rs2070106-AA carriers in the general population showed an age-dependent increase in frontotemporal white matter hyperintensities, suggestive of neuroinflammation in these regions. Together, these findings indicate that reduced CNP levels are associated with white matter tract inflammation and catatonic signs in humans.

Janova and colleagues next turned to a mouse model to investigate the underlying cellular mechanism of this myelin- 
A

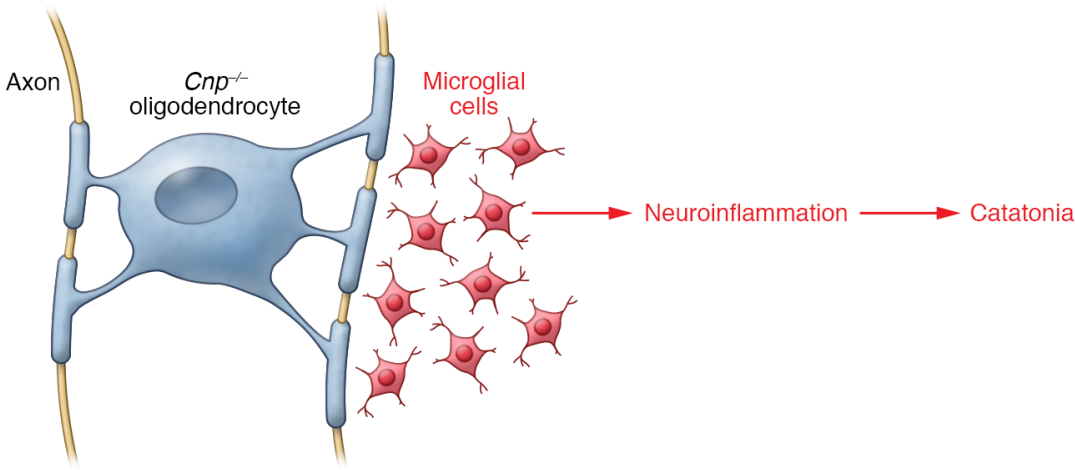

B

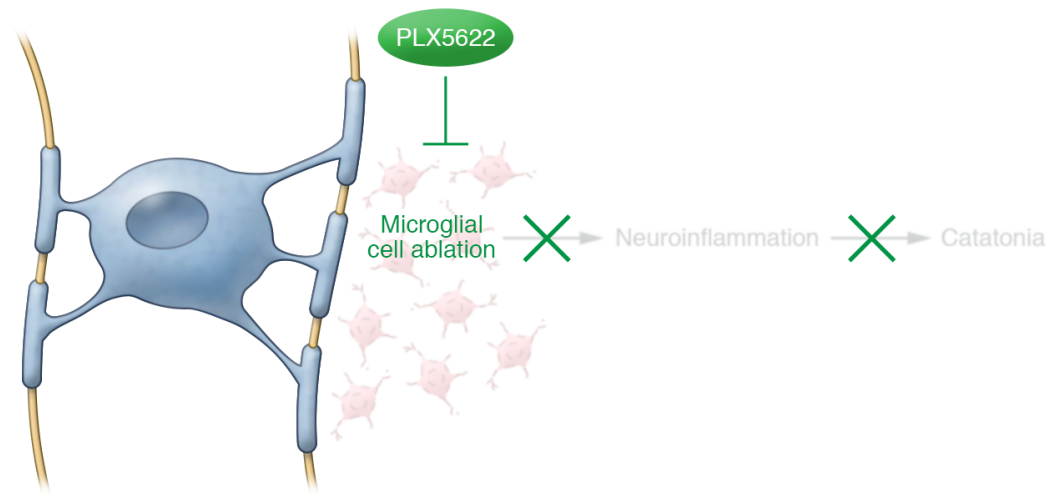

Figure 1. Microglial-mediated inflammation triggered by myelin deficits causes catatonic behavior. (A) In this issue, Janova et al. show that a minor myelin abnormality in mice lacking CNP causes microglial-mediated neuroinflammation and leads to catatonic symptoms. (B) Microglia ablation by the CSF1R inhibitor PLX5622 can both prevent and treat neuroinflammation and catatonia. associated catatonia (14). Young $\mathrm{Cnp}^{-/-}$ mice exhibited catatonic behavior in the absence of any gross motor deficits and had signs of both microglial activation and axon transport defects. The authors further investigated whether microglialmediated neuroinflammation causes catatonia in these mice by pharmacologically inhibiting colony-stimulating factor 1 receptor (CSF1R), which results in microglial depletion $(15,16)$. Excitingly, pretreatment of $\mathrm{Cnp}^{-/-}$mice with the CSF1R inhibitor PLX5622 prior to onset of catatonic behavior prevented development of this symptom (Figure 1). PLX5622 treatment also reduced microgliosis and axon transport defects, with these effects persisting up to 4 weeks after administration. This finding demonstrates a long-lasting antiinflammatory effect of microglial ablation. Janova and colleagues also used magnetic resonance spectroscopy to evaluate levels of the metabolite myo-inositol, a putative indicator of local neuroinflammation and microglial activation. Myo-inositol levels were elevated in white matter of $\mathrm{Cnp}^{-/-}$mice and further increased with age. Prophylactic microglial ablation was also sufficient to prevent elevated myoinositol levels in $\mathrm{Cnp}^{-/-}$mice, suggesting that microglial depletion prevents the progressive neuroinflammatory phenotype of these mice.

Importantly, microglial ablation was also sufficient to alleviate catatonic behavior in aging $\mathrm{Cnp}^{-/-}$mice already in an advanced state of neuroinflammation and neurodegeneration. Treatment of $\mathrm{Cnp}^{-/-}$mice with PLX5622 at 6 months of age also reduced microglial activation, axonal transport deficits, and astrocytosis in white matter. Finally, Janova et al. found that catatonic signs are a general phenotype of mice with mild myelin abnormalities and low-grade neuroinflammation, as these symptoms were also observed in mice heterozygous for $M b p$ and mice lacking myelin proteolipid protein $\left(P l p^{-/ y}\right)$.

\section{Summary and future directions}

By combining human data and mechanistic insight from the mouse, Janova and colleagues have described a cellular mechanism by which subtle myelin abnormalities lead to microglial-mediated inflammation and catatonic behavior (Figure 1). This study represents a notable advance, as it bridges the gap between correlation and causation for myelin impairment in a complex neuropsychiatric symptom. In addi- tion, Janova et al. have identified microglia as a promising cellular target for prevention and treatment of catatonia (14). However, it is important to note the potential effect of CSF1R inhibitors on nonmicroglial cells as a caveat to consider for this and future studies (17).

While Janova et al. (14) identify myelin-associated microgliosis as a cause of catatonic behavior, questions remain about how the observed pathological events fit together. What exactly is the putative myelin defect resulting from a CNP (or MBP or PLP) deficit that activates microglia, and how does such a defect result in microglia activation? Does the myelin defect directly trigger microglial inflammation, or does it do so indirectly, such as by inducing neurodegeneration? Janova et al. found that microglial ablation in $\mathrm{Cnp}^{-/-}$mice only partially prevented or ameliorated signs of neurodegeneration, suggesting that neurodegeneration may be at least partially independent of microglial activation (14). In addition, while $\mathrm{Cnp}^{-/-}$, $C n p^{+/-}$, and $P l p^{-1 y}$ mice develop neurodegenerative symptoms, $M b p^{+/-}$mice do not (13), yet all of these mice develop catatonia. Impaired axonal health may therefore not be the trigger for microglial activa- 
tion, as was previously hypothesized (11). However, it is possible that $M b p^{+/}$and other myelin-mutant mice develop subtle myelin-dependent changes in axonal function that cause microgliosis. Alternatively, the defect in myelin itself may directly trigger microglial activation. It will be important to determine whether microglial ablation can similarly prevent and treat catatonic symptoms in mice with other minor myelin deficits, such as those observed in $\mathrm{Mbp}^{+/-}$and $\mathrm{Pl} p^{-/ y}$ mice, and to use the commonalities between these models to identify the missing link between myelin and neuroinflammation.

The downstream mechanism by which neuroinflammation leads to catatonic behavior is also still unclear. Janova et al. speculate that white matter neuroinflammation may eventually impair synaptic circuit function (14). This could occur through multiple microglial-driven mechanisms, such as through release of neurotoxic ROS (18) or aberrant reactivation of complement cascade-mediated synaptic pruning (19-22). Notably, increased expression of the complement cascade component C4 has been correlated with schizophrenia in humans (22). Alternatively, signs of increased blood-brain barrier (BBB) permeability have also been correlated with schizophrenic symptoms (23). It will therefore also be important to investigate whether myelin-associated neuroinflammation compromises BBB integrity.

Interestingly, the $\mathrm{Cnp}^{+/-}$mouse exhibits behavioral abnormalities present in many neuropsychiatric diseases, including enhanced anxiety, depression, and impaired social and exploratory behavior (10). As white matter changes are associated with a wide range of disorders that affect higher-order processing, including schizophrenia, bipolar disorder, depression, autism, Huntington's disease, and Alzheimer's disease (2-4), future studies should investigate whether this myelinassociated neuroinflammation mecha- nism is generalizable to other symptoms of neuropsychiatric diseases. The findings by Janova et al. indicate a causal link between myelin deficits and catatonic behavior, suggesting that further investigation into this cellular mechanism could have farreaching implications for the study and treatment of neurological diseases.

\section{Acknowledgments}

This work was supported by the NIH/ National Institute of Neurological Disorders and Stroke (R01NS062796, R01NS097428, R01NS095889), the National Multiple Sclerosis Society (RG5203A4), and the Rachleff Family Endowment. We thank members of the Chan laboratory for insightful and helpful discussions and Lindsay Osso for her help with illustration of the figure.

Address correspondence to: Sarah E. Pease-Raissi, University of California, San Francisco, Sandler Neurosciences Center, 675 Nelson Rising Lane, Room 260, Box 3206, San Francisco, California 94158, USA. Phone: 415.353.4390; Email: sarah. raissi@ucsf.edu.

1. Philips T, Rothstein JD. Oligodendroglia: metabolic supporters of neurons. JClin Invest. 2017;127(9):3271-3280.

2. Nave KA, Ehrenreich H. Myelination and oligodendrocyte functions in psychiatric diseases. JAMA Psychiatry. 2014;71(5):582-584.

3. Filley CM, Fields RD. White matter and cognition: making the connection. J Neurophysiol. 2016;116(5):2093-2104.

4. Fields RD. White matter in learning, cognition and psychiatric disorders. Trends Neurosci. 2008;31(7):361-370.

5. Hakak Y, et al. Genome-wide expression analysis reveals dysregulation of myelination-related genes in chronic schizophrenia. Proc Natl Acad SciU S A. 2001;98(8):4746-4751.

6. Tkachev D, et al. Oligodendrocyte dysfunction in schizophrenia and bipolar disorder. Lancet. 2003;362(9386):798-805.

7. Mitkus SN, et al. Expression of oligodendrocyteassociated genes in dorsolateral prefrontal cortex of patients with schizophrenia. Schizophr Res. 2008;98(1-3):129-138.

8. Peirce TR, et al. Convergent evidence for 2',3'-cyclic nucleotide 3'-phosphodiesterase as a possible susceptibility gene for schizophrenia. Arch Gen Psychiatry. 2006;63(1):18-24.

9. Iwamoto K, Ueda J, Bundo M, Nakano Y, Kato T. Effect of a functional single nucleotide polymorphism in the $2^{\prime}, 3^{\prime}$-cyclic nucleotide $3^{\prime}$-phosphodiesterase gene on the expression of oligodendrocyte-related genes in schizophrenia. Psychiatry Clin Neurosci. 2008;62(1):103-108.

10. Hagemeyer $\mathrm{N}$, et al. A myelin gene causative of a catatonia-depression syndrome upon aging. EMBO Mol Med. 2012;4(6):528-539.

11. Lappe-Siefke C, et al. Disruption of Cnp1 uncouples oligodendroglial functions in axonal support and myelination. Nat Genet. 2003;33(3):366-374.

12. Wieser GL, et al. Neuroinflammation in white matter tracts of Cnp1 mutant mice amplified by a minor brain injury. Glia. 2013;61(6):869-880.

13. Poggi G, et al. Cortical network dysfunction caused by a subtle defect of myelination. Glia. 2016;64(11):2025-2040.

14. Janova H, et al. Microglia ablation alleviates myelin-associated catatonic signs in mice. J Clin Invest. 2018;128(2):734-745.

15. Waisman A, Ginhoux F, Greter M, Bruttger J. Homeostasis of microglia in the adult brain: review of novel microglia depletion systems. Trends Immunol. 2015;36(10):625-636.

16. Dagher NN, et al. Colony-stimulating factor 1 receptor inhibition prevents microglial plaque association and improves cognition in $3 \mathrm{xTg}-\mathrm{AD}$ mice. J Neuroinflammation. 2015;12:139.

17. Chitu V, Gokhan Ş, Nandi S, Mehler MF, Stanley ER. Emerging roles for CSF-1 receptor and its ligands in the nervous system. Trends Neurosci. 2016;39(6):378-393.

18. Block ML, Zecca L, Hong JS. Microglia-mediated neurotoxicity: uncovering the molecular mechanisms. Nat Rev Neurosci. 2007;8(1):57-69.

19. Stevens B, et al. The classical complement cascade mediates CNS synapse elimination. Cell. 2007;131(6):1164-1178.

20. Hong S, Dissing-Olesen L, Stevens B. New insights on the role of microglia in synaptic pruning in health and disease. Curr Opin Neurobiol. 2016;36:128-134.

21. Stephan AH, Barres BA, Stevens B. The complement system: an unexpected role in synaptic pruning during development and disease. Annu Rev Neurosci. 2012;35:369-389.

22. Sekar A, et al. Schizophrenia risk from complex variation of complement component 4. Nature. 2016;530(7589):177-183.

23. Schoknecht K, Shalev H. Blood-brain barrier dysfunction in brain diseases: clinical experience. Epilepsia. 2012;53(suppl 6):7-13. 